\title{
Classification-Segmentation Pipeline for MRI via Transfer Learning and Residual Networks
}

\author{
Nghia Duong-Trung $\bowtie$ \\ Technische Universität Berlin \\ Straße des 17. Juni 135, 10623 Berlin, Germany \\ Email: nghia.duong-trung@tu-berlin.de
}

\author{
Dung Ngoc Le Ha \\ Can Tho University of Technology \\ 74000 Can Tho city, Vietnam \\ Email: hlndung@ctuet.edu.vn
}

\author{
Hiep Xuan Huynh \\ Can Tho University \\ 74000 Can Tho city, Vietnam \\ Email: hxhiep@ctu.edu.vn
}

\begin{abstract}
Artificial intelligence association into brain magnetic resonance imaging (MRI) and clinical practices embrace substantial cancer diagnosis improvement. The advancement of deep learning has improved the processing and analysis of MRI, boosting models' performance, decreasing the destructive effects of data sources overload, and increasing accurate detection and time efficacy. However, that specific dataset leads to diverse research fields such as image processing and analysis, detection, registration, segmentation, and classification. This paper proposes a decision-making pipeline for MRI data by combining image classification and segmentation. First, the pipeline should correctly produce a correct decision given an MRI image. If the figure is classified as defective, the pipeline can extract defect regions and highlight them accordingly. We have implemented several advanced convolutional neural networks with transfer learning and residual techniques to address two broad clinical concerns in one decision-making workflow.
\end{abstract}

\section{INTRODUCTION AND MOTIVATION}

$\mathbf{T}$ ODAY, clinical practice is an area of interest and research where extensive research and technical recommendations have been developed in response to increasingly complex challenges [1], [2], [3]. Identifying and analyzing diseases is increasingly difficult because they are ever more sophisticated. Fortunately, artificial intelligence has revolutionized clinical practice in many areas such as cancer diagnosis with medical imaging [4], automatic classification diseases based on descriptions [5], [6], and maximizing hospital efficiency [7]. Among many approaches, deep learning has been proven superior in a wide range of clinical data and practice scenarios. Regarding MRI, the complex feature can be represented effectively by utilizing deep learning-based models in detection, registration, classification, and segmentation problems.

Employing a convolutional neural network $(\mathrm{CNN})$ for image classification is one of the reasonable rises, and it is an essential model in developing an automatic disease diagnosis [8], [9]. Among competitors of the ImageNet challenge in 2012, the deep learning-based model AlexNet proposed by Krizhevsky et al.[10] won the championship. CNN has become the backbone architecture for addressing almost all problems in computer science. Many CNN-based approaches have been investigated for addressing MRI image classification [11].

Due to CNN's dominant performance in the MRI classification domain, people began exploiting CNN for MRI segmentation. More specifically, MRI diagnosis is the subdivision of different brain regions to detect brain diseases, such as cancer and Parkinson's syndrome. Consequently, automatic segmentation of defect regions in MRI is significantly essential in everyday clinical routines and medical research [12]. With the performance of $\mathrm{CNN}$, excellent segmentation approaches have been developed based on CNN and continuously become front tier in particular segmentation competitions [13].

However, one interesting research question that someone might consider is that we should combine several research domains and develop a practical workflow that supports medical analysis and recommendation. This article aims to propose a decision-making pipeline for MRI data by combining image classification and image segmentation. First, given an MRI image, the classification part of the pipeline should make a correct decision. If the brain MRI is classified as defective, the segmentation part can extract defect regions and highlight them accordingly. Thus, the Class-Seg workflow is designed and implemented by leveraging transfer learning, residual network [14] design, and several state-of-the-art CNN models.

\section{BLUEPRINT OF THE MRI Class-SEG PIPELINE}

As mentioned in the previous section, we propose a machine learning pipeline to support MRI diagnosis by (i) applying transfer learning techniques to select the best detection model and (ii) integrating classification with segmentation in one channel to improve MRI diagnosis and treatment. The blueprint of our proposed pipeline is presented in Figure 1. Here, the MRI dataset is randomly divided without replacement into several portions. Five primarily used CNN models are selected for the task of classification. Technically, the CNN part's weights are transferred from pre-trained models on ImageNet. The CNN part is freezing out of the backpropagation process. Then it is flattened and fed into our proposed dense layers, see Figure 2, where the weights are learned. The authors implement ResUNet based on UNet architecture comprising several residual blocks to overcome the vanishing gradients problems in deep CNN architecture regarding the segmentation task. We present how classification and segmentation tasks can be combined in Algorithm 1. Note in the pseudo-code that phases 1 and 2 correspond to points (i) and (ii) in this section, respectively. Transfer learning is applied in phase 1 , where multiple models are reused, while in phase 2 , we train the segmentation model from scratch. 


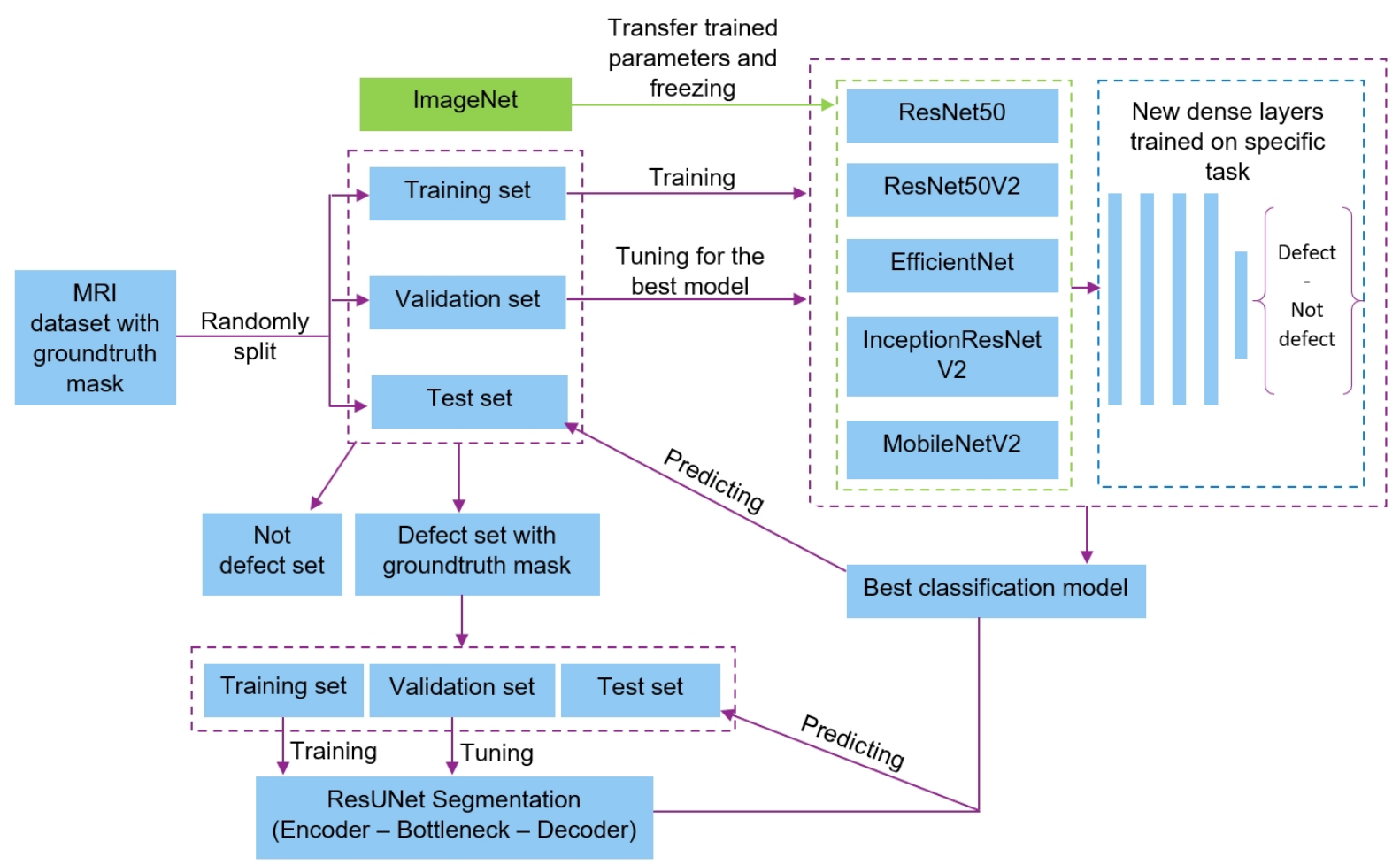

Figure 1. The Proposed Classification-Segmentation Pipeline for Brain MRI.

\begin{tabular}{|c|c|c|}
\hline Layer (type) & Output Shape & Param \# \\
\hline dense (Dense) & (None, 1024) & (from flatten) \\
\hline dropout (Dropout) & (None, 1024) & 0 \\
\hline dense (Dense) & (None, 1024) & 1049600 \\
\hline dropout (Dropout) & (None, 1024) & 0 \\
\hline dense (Dense) & (None, 1024) & 1049600 \\
\hline dropout (Dropout) & (None, 1024) & 0 \\
\hline dense (Dense) & (None, 256) & 262400 \\
\hline dropout (Dropout) & (None, 256) & 0 \\
\hline dense (Dense) & (None, 2) & 514 \\
\hline
\end{tabular}

Figure 2. Our proposed dense layers to be trained with MRI dataset.

\section{TRANSFER LEARNING}

Transfer learning technique is to reuse the pre-trained models learned in one or more different domains and utilize the knowledge to enhance learning in any other domain [15], [16]. Reusing a trained model that solves a problem similar to your data source is very versatile. It allows a machine learning approach to be applied to data drawn from a wide range of different sources from the one upon which it has initially been trained [17], [18], [19]. It might take weeks to train modern CNN models with millions of parameters fully. Transfer learning proposed many re-trained options such as fine-tuning model weight, freezing layers, and even re-train from scratch. It shortcuts a lot of network design and training by transferring a trained set of parameters from a predefined category and re-weights the model's parameters from new data. This technique, called inductive transfer learning, aims to effectively improve training in the target domain by practicing knowledge transferability from many other sources. By reupdating weights, the effects of dissimilar observations will be reduced, and it might thus produce a more objective approach. Every CNN-based model consists of two parts, a series of convolutional-pooling layers as the tail and sequences of dense layers as the head. One transferability strategy is to transfer the learned parameters to the tail and freeze it. Flatten is applied at the tail's last layer before integrating it into the head. Backpropagation and parameters update is done within the head. In Figure 3, we present the key difference between traditional machine learning and the adoption of transfer learning.

\section{EXPERIMENTS}

\section{A. Experimental MRI Dataset}

We have exploited our proposed classification-segmentation pipeline on the most reputable brain MRI segmentation dataset $^{1}$. By the time we conducted this paper, there had been 42 code solutions and six discussions on the dataset. However, none developed a systematic pipeline for the dual task of binary classification and image segmentation. The 1GB contains 7860 brain MRI figures manually annotated with fluid-attenuated inversion recovery (FLAIR) abnormality

\footnotetext{
${ }^{1}$ https://www.kaggle.com/mateuszbuda/lgg-mri-segmentation
} 


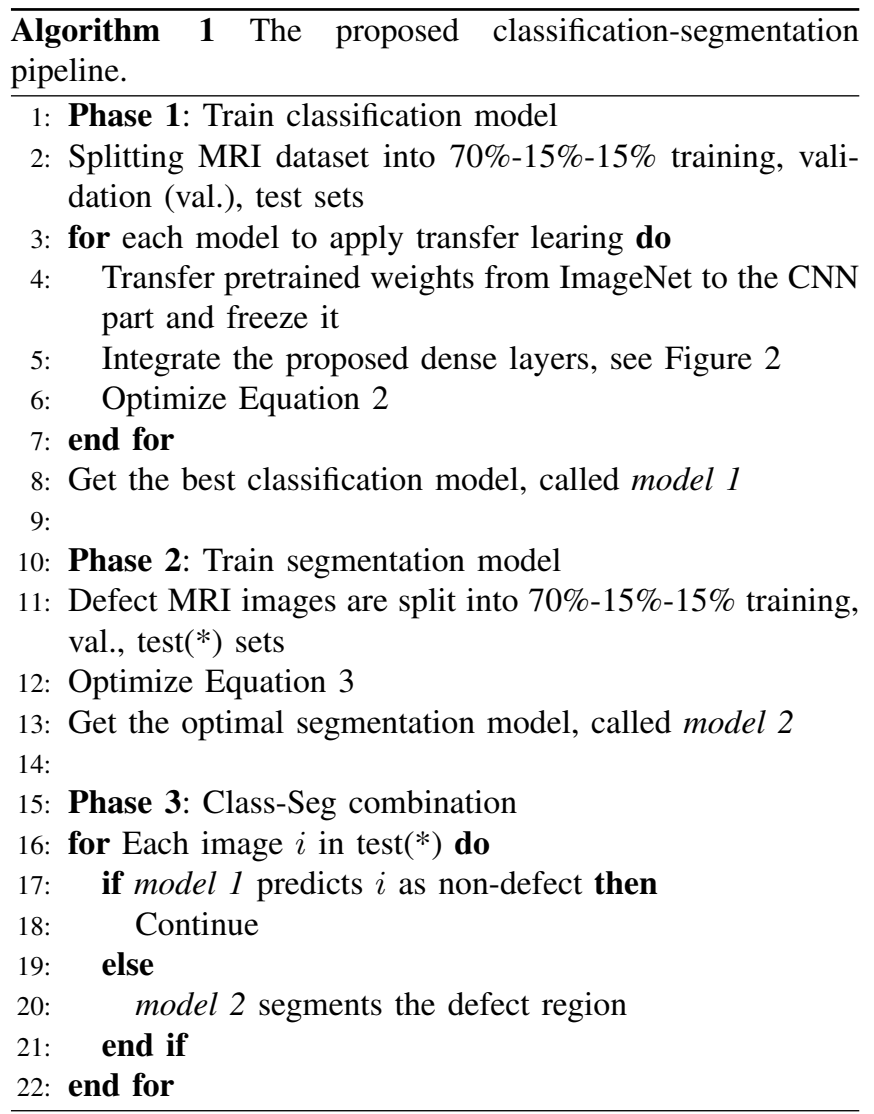

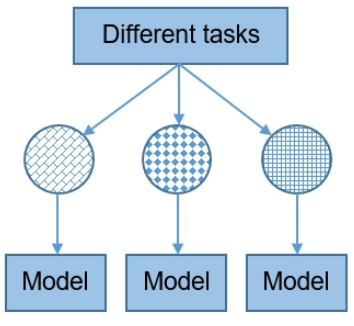

a) traditional machine learning

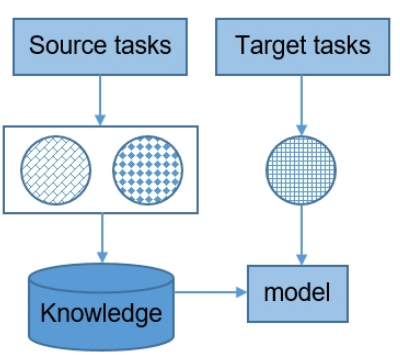

b) transfer learning
Figure 3. In a), different models for seperated tasks. In b) one apporach can be re-used for many tasks.

segmentation masks. First, the dataset was collected from 110 patients included in The Cancer Genome Atlas lower-grade glioma collection. Then, the images with at least one possible FLAIR sequence and genomic cluster are selected.

\section{B. Loss Functions}

In the previous section, the authors have mentioned that the pipeline consists of two distinct tasks: first, the model should correctly classify defective images from normal ones; second, after the image is classified as defected, it is fed into segmentation to reveal the region of abnormality. Hence, the pipeline applies two different loss functions: categorical crossentropy loss to address binary classification scenario and Focal
Tversky [20] loss which is highly recommended for handling imbalanced data and small regions-of-interest segmentation.

Categorical cross-entropy (CCE) loss is a softmax activation plus a cross-entropy (CE) loss. The CE is defined as follows in a binary classification problem.

$$
\mathrm{CE}=\sum_{i=1}^{C=2} y_{i} \log \left(s_{i}\right)=-y_{1} \log \left(s_{1}\right)-\left(1-y_{1}\right) \log \left(1-s_{1}\right),
$$

where $y_{i}, s_{i}$, and $C$ are the groundtruth, the predicted $\mathrm{CNN}$ score and the number of class respectively. Then CE plus softmax activation which yields CCE as follows.

$$
\mathrm{CCE}=-\sum_{i}^{C} y_{i} \log \left(f(s)_{i}\right),
$$

where $f(s)_{i}=\frac{e^{s_{i}}}{\sum_{j}^{C} e^{s_{j}}}$.

The focal Tversky (FT) loss is defined as follows.

$$
\mathrm{FT}=\sum_{i}^{C}\left(1-\mathrm{T}_{i}\right)^{\frac{1}{\gamma}}
$$

where $\gamma=\frac{4}{3}$ by default as described in [20]. $\mathrm{T}_{i}$ is denoted as follows.

$$
\mathrm{T}_{i}=\frac{\sum_{j=1}^{N} p_{j i} g_{j i}+\epsilon}{\sum_{j=1}^{N} p_{j i} g_{j i}+\alpha \sum_{j=1}^{N} p_{j \bar{i}} g_{j i}+\beta \sum_{j=1}^{N} p_{j i} g_{j \bar{i}}+\epsilon},
$$

where $N$ provides the total number of pixels in an image. $\epsilon$ is numerical stability. $p_{j i}$ is the probability that pixel $j$ is of the lesion class $i$, while $p_{j \bar{i}}$ is that of non-lesion class $i$. The same meaning is applied to $g_{j i}$ and $g_{j \bar{i}} . \alpha$ and $\beta$ are tunable hyperparameters to shift the recall emphasis in case of large class imbalance. In our experiments, we set the value of $\alpha=0.7$ and $\beta=0.75$.

\section{Experimental Results}

For the task of classification, the authors have deployed 5 well-known CNN models in computer vision community, e.g. ResNet50 [21], ResNet50V2 [21], InceptionResNetV2 [22], EfficientNet [23], and MobileNetV2 [24]. These models are easily called using TensorFlow API [25]. While in the segmentation task, the authors implement ResUNet [26], one of the most state-of-the-art segmentation models. The authors run three times for each approach and report the average scores. The classification and segmentation performance of all models have presented in Table I. We illustrate several segmentation samples in Figure 4.

In Table I, the first five models are used to select the best candidate for the classification task. The best solution is the InceptionResNetV2 model, which achieves $95 \%$ of accuracy. The authors report the number of trainable parameters, the average training duration, the accuracy score on the test set, and two basic F1-score schemes [27]. Turning to segmentation, 
Table I

Classification and Segmentation Performance of all Models

\begin{tabular}{|l|c|c|c|c|c|}
\hline \multicolumn{1}{|c|}{ Model } & $\begin{array}{c}\text { Trainable params } \\
\text { (million) }\end{array}$ & $\begin{array}{c}\text { Training time } \\
\text { (minutes) }\end{array}$ & Test Acc. & $\begin{array}{c}\text { Micro avg } \\
\text { F1-score }\end{array}$ & $\begin{array}{c}\text { Macro avg } \\
\text { F1-score }\end{array}$ \\
\hline ResNet50 & 59.452 .162 & 15.04 & 0.93 & 0.93 & 0.92 \\
\hline ResNet50V2 & 59.436 .930 & 13.36 & 0.88 & 0.88 & 0.86 \\
\hline InceptionResNetV2 & 70.795 .106 & 27.78 & $\mathbf{0 . 9 5}$ & $\mathbf{0 . 9 5}$ & $\mathbf{0 . 9 5}$ \\
\hline EfficientNetB0 & 27.342 .206 & 13.48 & 0.66 & 0.67 & 0.43 \\
\hline MobileNetV2 & 25.558 .530 & 09.33 & 0.41 & 0.42 & 0.37 \\
\hline \hline & & & Val. Tversky & & \\
\hline ResUNet & 1.206 .129 & 0.63 & 0.89 & - & - \\
\hline
\end{tabular}
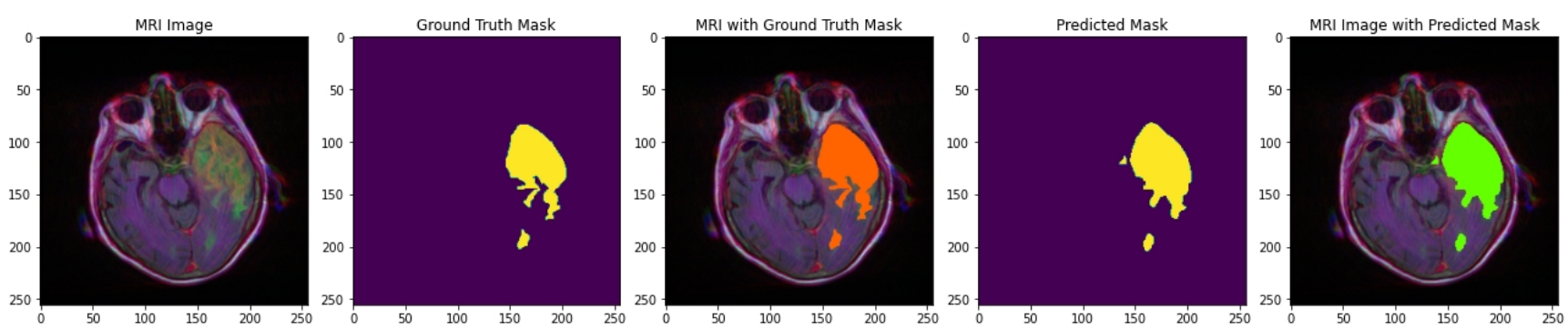

MRI Image

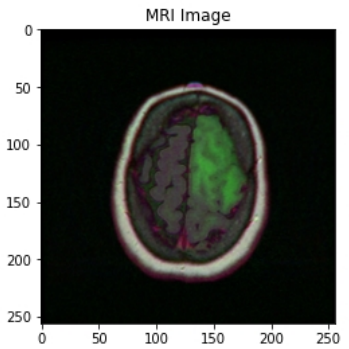

Ground Truth Mask

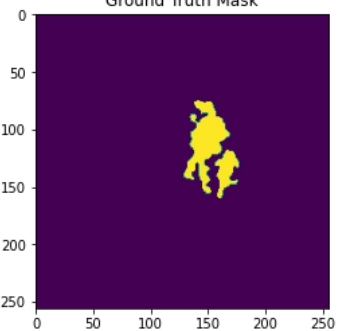

MRI with Ground Truth Mask

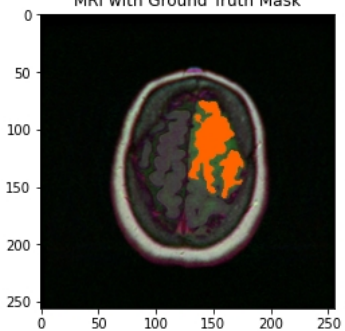

Predicted Mask

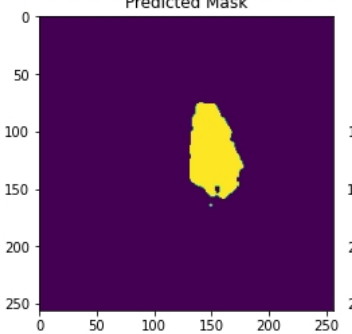

MRI Image with Predicted Mask

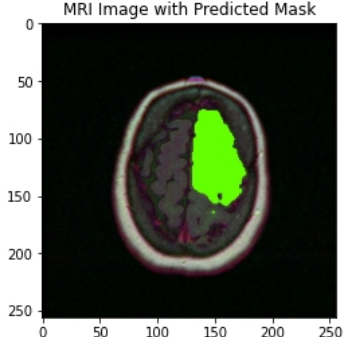

Figure 4. The Result of the Segmentation Process. From left to right in each row, the original MRI figure, annotated groundtruth mask on defect region, the overlapping of them, the predicted mask provided by the segmentation process, and the predicted overlapping.

the authors implement ResUNet because of the speed, high performance.We report the Tversky score for this task.

\section{Reproducibility}

The authors have conducted all experiments on Google Colab. GPU runtime type has been activated by default. We encourage further reproducibility by engaging readers by revealing all models' weights and architecture. Regarding the paper's length restriction, the authors add additional materials and other experiment resources in our GitHub repository ${ }^{2}$.

\section{CONCLUSiOn}

We have described and implemented the proposed ClassSeg workflow by leveraging transfer learning and residual network together with several state-of-the-art convolutional neural models. We aim to combine the classification and segmentation of brain MRI into a single clinical practice. To our knowledge, we are the first to combine two research

\footnotetext{
${ }^{2}$ https://github.com/duongtrung/Class-Seg-Brain-MRI
}

directions on the well-known Kaggle brain MRI dataset, in which more than 40 code solutions have been investigated. Intensive experiments have been conducted to develop a clinically acceptable automatic workflow for better brain MRI diagnosis.

\section{REFERENCES}

[1] L. da Cruz, C. Sierra-Franco, G. Silva-Calpa, and A. Raposo, "Enabling autonomous medical image data annotation: A human-in-the-loop reinforcement learning approach," in Proceedings of the 16th Conference on Computer Science and Intelligence Systems, ser. Annals of Computer Science and Information Systems, M. Ganzha, L. Maciaszek, M. Paprzycki, and D. Ślęzak, Eds., vol. 25. IEEE, 2021, p. 271-279.

[2] J. Dörpinghaus, S. Schaaf, V. Weil, and T. Hübenthal, "An efficient approach towards the generation and analysis of interoperable clinical data in a knowledge graph," in Proceedings of the 16th Conference on Computer Science and Intelligence Systems, ser. Annals of Computer Science and Information Systems, M. Ganzha, L. Maciaszek, M. Paprzycki, and D. Ślęzak, Eds., vol. 25. IEEE, 2021, p. 59-68.

[3] R. Damaševičius, O. Abayomi-Alli, R. Maskeliūnas, and A. AbayomiAlli, "Bilstm with data augmentation using interpolation methods to improve early detection of parkinson disease," in Proceedings of the 2020 Federated Conference on Computer Science and Information 
Systems, ser. Annals of Computer Science and Information Systems, S. Agarwal, D. N. Barrell, and V. K. Solanki, Eds. IEEE, 2020, pp. 371-380.

[4] M. L. Giger, "Machine learning in medical imaging," Journal of the American College of Radiology, vol. 15, no. 3, pp. 512-520, 2018.

[5] N. Duong-Trung, X. N. Hoang, T. B. T. Tu, K. N. Minh, V. U. Tran, and T.-D. Luu, "Blueprinting the workflow of medical diagnosis through the lens of machine learning perspective," in 2019 International Conference on Advanced Computing and Applications (ACOMP). IEEE, 2019, pp. 23-26.

[6] N. Duong-trung, N. Quynh, T. Tang, and X. S. Ha, "Interpretation of Machine Learning Models for Medical Diagnosis," Advances in Science, Technology and Engineering Systems Journal, vol. 5, no. 5, pp. 469-477, 2020.

[7] M. Fairley, D. Scheinker, and M. L. Brandeau, "Improving the efficiency of the operating room environment with an optimization and machine learning model," Health care management science, vol. 22, no. 4, pp. 756-767, 2019.

[8] H. Benbrahim, H. Hachimi, and A. Amine, "Deep convolutional neural network with tensorflow and keras to classify skin cancer images," Scalable Computing: Practice and Experience, vol. 21, no. 3, pp. 379$390,2020$.

[9] S. Kusuma and J. D. Udayan, "Analysis on deep learning methods for ecg based cardiovascular disease prediction," Scalable Computing: Practice and Experience, vol. 21, no. 1, pp. 127-136, 2020.

[10] A. Krizhevsky, I. Sutskever, and G. E. Hinton, "Imagenet classification with deep convolutional neural networks," Advances in neural information processing systems, vol. 25, pp. 1097-1105, 2012.

[11] T. Kaur and T. K. Gandhi, "Deep convolutional neural networks with transfer learning for automated brain image classification," Machine Vision and Applications, vol. 31, no. 3, pp. 1-16, 2020.

[12] M. Mittal, M. Arora, T. Pandey, and L. M. Goyal, "Image segmentation using deep learning techniques in medical images," in Advancement of machine intelligence in interactive medical image analysis. Springer, 2020, pp. 41-63.

[13] Z. Akkus, A. Galimzianova, A. Hoogi, D. L. Rubin, and B. J. Erickson, "Deep learning for brain mri segmentation: state of the art and future directions," Journal of digital imaging, vol. 30, no. 4, pp. 449-459, 2017.

[14] G. Liang and L. Zheng, "A transfer learning method with deep residual network for pediatric pneumonia diagnosis," Computer methods and programs in biomedicine, vol. 187, p. 104964, 2020.

[15] W. Ying, Y. Zhang, J. Huang, and Q. Yang, "Transfer learning via learning to transfer," in International Conference on Machine Learning. PMLR, 2018, pp. 5085-5094.

[16] Q. Yang, Y. Zhang, W. Dai, and S. J. Pan, Transfer learning. Cambridge University Press, 2020.

[17] N. Duong-Trung, L.-D. Quach, and C.-N. Nguyen, "Learning deep transferability for several agricultural classification problems," International Journal of Advanced Computer Science and Applications, vol. 10, no. 1, 2019.

[18] N. Duong-Trung, L.-D. Quach, M.-H. Nguyen, and C.-N. Nguyen, “A combination of transfer learning and deep learning for medicinal plant classification," in Proceedings of the 2019 4th International Conference on Intelligent Information Technology, 2019, pp. 83-90.

[19] _ _ "Classification of grain discoloration via transfer learning and convolutional neural networks," in Proceedings of the 3rd International Conference on Machine Learning and Soft Computing, 2019, pp. 27-32.

[20] N. Abraham and N. M. Khan, "A novel focal tversky loss function with improved attention u-net for lesion segmentation," in 2019 IEEE 16th International Symposium on Biomedical Imaging (ISBI 2019). IEEE 2019, pp. 683-687.

[21] K. He, X. Zhang, S. Ren, and J. Sun, "Deep residual learning for image recognition," in Proceedings of the IEEE conference on computer vision and pattern recognition, 2016, pp. 770-778.

[22] C. Szegedy, S. Ioffe, V. Vanhoucke, and A. Alemi, "Inception-v4, inception-resnet and the impact of residual connections on learning," in Proceedings of the AAAI Conference on Artificial Intelligence, vol. 31, no. 1, 2017.

[23] M. Tan and Q. Le, "Efficientnet: Rethinking model scaling for convolutional neural networks," in International Conference on Machine Learning. PMLR, 2019, pp. 6105-6114.

[24] M. Sandler, A. Howard, M. Zhu, A. Zhmoginov, and L.-C. Chen, "Mobilenetv2: Inverted residuals and linear bottlenecks," in Proceedings of the IEEE conference on computer vision and pattern recognition, 2018, pp. 4510-4520.

[25] D. Sarkar, R. Bali, and T. Ghosh, Hands-On Transfer Learning with Python: Implement advanced deep learning and neural network models using TensorFlow and Keras. Packt Publishing Ltd, 2018.

[26] F. I. Diakogiannis, F. Waldner, P. Caccetta, and C. Wu, "Resunet-a: a deep learning framework for semantic segmentation of remotely sensed data," ISPRS Journal of Photogrammetry and Remote Sensing, vol. 162, pp. 94-114, 2020.

[27] N. Duong-Trung, Social Media Learning: Novel Text Analytics for Geolocation and Topic Modeling. Cuvillier Verlag, 2017. 